\title{
A preliminary investigation of the AUDIT and DUDIT in comparison to biomarkers for alcohol and drug use among HIV-infected clinic attendees in Cape Town, South Africa
}

\author{
R Kader', S Seedat², JR Koch ${ }^{3}$, CD Parry',2 \\ ${ }^{1}$ Alcohol \& Drug Abuse Research Unit, Medical Research Council, South Africa \\ 2Department of Psychiatry, Stellenbosch University, South Africa \\ ${ }^{3}$ Institute for Drug and Alcohol Studies, Virginia Commonwealth University, USA
}

\begin{abstract}
Objective: There is growing concern about the effect of substance use on HIV treatment outcomes. The study objectives included: (i) evaluating whether the use of validated questionnaires (AUDIT and DUDIT) provide useful and consistent information of alcohol and drug consumption when compared with the use of biomarkers of alcohol in (urine and hair) and drugs in (urine) and (ii) assessing the feasibility of using self-report measures compared with urine and hair tests. Method: Participants were HIV positive patients attending an HIV community health clinic in Kraaifontein, Cape Town. Hair and urine samples were collected and analysed for alcohol, in Fatty Acid Ethyl Esters (FAEE) and in Ethyl Glucuronide and (EtG), and drugs. Biological markers were compared with self-report measures of alcohol and drug consumption in terms of sensitivity, specificity. Forty-three participants completed the self-report measures, while 30 provided hair and urine samples. Results: On the AUDIT, 18 (41.9\%) participants screened positive for harmful and hazardous drinking and 13 (30.2\%) participants on the DUDIT screened positive for having a drug-related problem. Two of 30 participants (7\%) tested positive for alcohol abuse on FAEE analysis. For EtG, 6 of 24 (25\%) participants tested positive for alcohol abuse. On hair drug analysis, all 30 participants tested negative for cannabis, amphetamines, opiates, cocaine, PCP and methaqualone. On the urinalysis, lof 30 participants tested positive for cannabis and everyone tested negative for all other drugs included in the screening. Conclusion: Substance use among patients attending HIV clinics appears to be a problem, especially alcohol. Self-report measures seem to be a more cost effective option for screening of alcohol and drug abuse in resource poor settings.
\end{abstract}

Keywords: Biological markers; Substance abuse; HIV; South Africa

Received: $25-01-2011$

Accepted: $16-08-2011$

doi: http://dx.doi.org/10.4314/ajpsy.v15i5.43

\section{Introduction}

In South Africa, the overall prevalence rate for HIV is estimated to be $10.6 \%$, with $17 \%$ of all persons between (15-49 years) being HIV positive. Currently, there are 5.2 million people living with HIV and the number of new infections estimated for 2009 was $413000 .{ }^{1}$ Although alcohol surpasses other drugs as the most abused substance in South Africa, in recent years there has been an increase in the use of heroin, crack cocaine and amphetamine-type stimulants. ${ }^{2}$ This country had the 2 nd highest prevalence of past year substance abuse (5.8\%) compared to the 14 other participant countries in the World Mental Health Survey. ${ }^{3}$ According to the South African National HIV, Incidence, Behaviour and Communication Survey of 2008, $41.5 \%$ men and 17.1\% women reported current alcohol use while $17 \%$ men and $2.9 \%$ women reported risky or hazardous and harmful drinking. ${ }^{4}$

Given the relationship between substance use and HIV infection, it is likely that many infected persons are also 
substance abusers. Substance abuse by persons living with HIV and AIDS (PLWHA) can lead to non-adherence to treatment, especially anti-retroviral therapy ${ }^{5}$ PLWHA who have problematic substance use are also more susceptible to AIDSrelated opportunistic infections and sub-optimal health compared to those who do not use substances ${ }^{6}$ Despite this, there has been scant investigation of the relationship between substance abuse and HIV among PLWHA in South Africa. One study conducted among a convenience sample of 149 HIV positive patients receiving medical care at an infectious disease clinic in Cape Town found that $10.1 \%$ of the patients were alcohol dependent, with $22.7 \%$ of males reporting alcohol dependence compared to $4.7 \%$ of females. ${ }^{7}$ A more recent study, also conducted in Cape Town, found that of the 465 HIV positive patients enrolled for HIV treatment and care at primary health care clinics, 7\% reported alcohol dependence/abuse. ${ }^{8}$

Often with stigmatized behaviours such as substance use, honesty is especially important. ${ }^{9}$ It seems that most patients attending HIV clinics will not talk about their substance use behaviour owing to stigmatization or fear of not being given antiretrovirals (ARVs). ${ }^{10}$ Untreated substance abuse may result in HIV treatment being ineffective secondary to compromised adherence and ongoing risk behavior which may result in reinfection. CD4 counts may also be reduced, drug resistance may occur and this may result in an earlier onset of death. ${ }^{11}$ Health care professionals may also choose not to confront alcohol and drug-related behaviour and associated problems in patients due to their own negative attitudes towards alcohol and drug use. ${ }^{12}$ However, objective testing of alcohol and drug usage may assist in identifying problematic drug and alcohol use early so that they may be addressed on an individual as well as a community level which, in turn, may improve treatment outcomes. ${ }^{12,13}$

There is increasing recognition that self-report measures of alcohol and drug use can be useful aids in primary health care setting $s^{14}$, but less is known about the accuracy of using selfreport measures in HIV clinics. Patients with HIV may harbour fears that being open about their substance use may result in stigmatization by health workers and may compromise their eligibility to receive ARVs. Biological markers such as Fatty Acid Ethyl Esters (FAEE) and Ethyl Glucuronide (EtG) when used in addition to self-report questionnaires, such as the Alcohol Use Disorders Identification Test (AUDIT) and Drug Use Disorders Identification Tool (DUDIT), could assist in the verification of self-reported drug and alcohol abuse. ${ }^{14}$

FAEE and EtG are metabolites of ethanol and are very specific biomarkers for alcohol. Both FAEE and EtG are deposited in the hair, enabling a longer window of opportunity for diagnosis of alcohol consumption..$^{15}$ Analysis of these metabolites in hair and urine can be conducted with gas chromatography/mass spectrometry (GC/MS) to identify and quantify markers indicative of alcohol consumption. ${ }^{15,16}$ The analysis of urine for drug detection also uses the GC/MS technique and the HPLC/MS/MS technique can be used for detecting drugs in hair. ${ }^{16}$ This pilot study aimed to assess the relative utility of the AUDIT and DUDIT screening too ls compared with selected biomarkers for alcohol and drug use disorders in HIV clinics. Specific objectives included: (i) evaluating whether the use of validated questionnaires [AUDIT and DUDIT] provide useful and consistent information when compared with biomarkers of alcohol and drug consumption in urine (drugs) and hair (alcohol and drugs) and (ii) assessing the feasibility (cost and practicality) of using selfreport measures compared with urine and hair tests in HIV clinic populations.

\section{Method}

\section{Design}

This pilot study used a cross-sectional research design.

\section{Setting and participants}

Data were collected in February and March 2010 at the Wallacedene Community Health Clinic (CHC) in Kraaifontein, Cape Town. The clinic is situated in one of the poorest communities in Cape Town, an informal settlement established between 1985 and 1989, with very high rates of HIV, TB, gangrelated crime and alcohol and drug use. The clinic population is predominantly Coloured (mixed race) and "Black African" and the clinic lacks basic resources, including not having enough rooms in which to attend to patients. HIV patients are attended to in a converted shipping container which serves as a clinic room. Often there is not enough space to accommodate all patients and they have to queue outside. The clinic provides HIV care, antiretroviral therapy, tuberculosis and other health care services free of charge. Study participants were HIV positive patients who were 18 years and older, with 28 receiving antiretroviral therapy. Forty-three patients were recruited into the study. All 43 completed the self-report measures for alcohol and drug use (see below) and demographic data was obtained on all 43 patients. However, due to budgetary constraints, urine and hair samples were only obtained from the first 30 of the 43 patients that were willing to give their hair and urine samples.

\section{Study procedures}

A semi-structured diagnostic interview questionnaire (Mini International Neuropsychiatric Interview) was administered in English, Afrikaans and Xhosa and several self-report measures were completed. ${ }^{17}$ The interviews were administered by trained interviewers who had experience working within disadvantaged communities. The interviews were conducted in a school hall situated near the clinic, which afforded patients privacy for interviewing and collection of urine and hair samples. Participants were interviewed for approximately 45 minutes, following which urine and hair samples were collected in a settling that allowed for privacy. Hair was cut approximately $1 \mathrm{~mm}$ from the scalp and then wrapped into foil with the root end marked. The hair sample was sealed in an envelope with a barcode. Samples were sent to Trimega Laboratories Limited in the United Kingdom to be analysed. At the time of this study, hair testing kits were not available in South Africa and laboratories did not have the necessary equipment to analyse hair samples. The urine samples were collected in a $50 \mathrm{ml}$ container onsite which was then stored in a cooler box and subsequently sent to a laboratory in Pretoria, South Africa for analysis.

The decision to use hair analysis, a relatively expensive biomarker test, as compared to cheaper blood and urine analyses for the detection of alcohol and drug use was guided by several factors: Firstly, hair analysis allows for the detection of drugs or alcohol over a longer period (up to 12 months) 
depending on the length of the hair strand. ${ }^{15}$ In contrast, blood and urine samples have a window of detection of several days to 2-3 weeks only. Secondly, hair testing is considered the gold standard of biomarkers and has been shown to have higher levels of sensitivity and specificity for alcohol/drug detection than blood and urine tests. ${ }^{16}$ Thirdly, we were interested in qualitatively assessing the feasibility and cultural acceptability of collecting hair samples in this community.

Completed questionnaires were checked every day, entered into an Excel spreadsheet and then exported to SPSS for cleaning and analysis. Participants received a voucher of R50 for their participation. Ethical approval to conduct this research was provided by the Health Research Ethics Committee of the University of Stellenbosch, Cape Town. Written informed consent was obtained from all participants.

\section{Assessment}

Sociodemographic and clinical data

Demographic questions included age, gender, race, marital status, highest level of education attained, level of income and employment status. Clinical data included date of diagnosis of HIV and receipt of antiretroviral therapy (yes/no).

\section{The Alcohol Use Disorders Identification Test (AUDIT)}

The AUDIT screens for hazardous and harmful patterns of alcohol use and alcohol dependence. It is a 10-item, self-rating questionnaire developed by the World Health Organisation, and internationally validated for use in primary health and community settings. A total score of 8 or more on the AUDIT indicates hazardous and harmful alcohol use as well as possible alcohol dependence. ${ }^{14}$

\section{The Drug Use Disorders Identification Test (DUDIT)}

The DUDIT is a screening instrument used to identify patterns of drug use and drug related problems (i.e., substance abuse/harmful use or dependence). It consists of 11 items, and includes a list of different drugs. Drug-related problems are indicated by a score of 6 or more for men and a score of 2 or more for women. A score of 25 or more, for both sexes, is indicative of a high probability of drug dependence. ${ }^{18}$

\section{Urinalysis}

Urine was tested for cannabis, amphetamines, opiates, cocaine, phencyclidine (PCP) and methaqualone, using the following cut-offs: $15 \mathrm{ng} / \mathrm{mL}$ for cannabis, $500 \mathrm{ng} / \mathrm{mL}$ for amphetamines $2000 \mathrm{ng} / \mathrm{mL}$ for opiates, $150 \mathrm{ng} / \mathrm{mL}$ for cocaine, $25 \mathrm{ng} / \mathrm{mL}$ for PCP and $300 \mathrm{ng} / \mathrm{mL}$ for methaqualone.

\section{Hair alcohol analysis}

A hair sample of approximately $3 \mathrm{~cm}$ length was taken from each participant to enable a 3 month diagnosis of alcohol consumption. Hair analysis was conducted using GC/MS technique.

Fatty Acid Ethyl Esters (FAEE) \& Ethyl glucuronide (EtG) FAEE is a metabolite of ethanol and indicates the amount of alcohol consumed. FAEE is derived from a range of fatty acids secreted in the body. The following cut-offs were applied: teetotallers (less than $0.20 \mathrm{ng} / \mathrm{mg}$ ), questionable alcohol use (0.21-0.50 ng/mg), lower cut-off (0.51-0.99) and higher cut-off (1.00ng/mg and above). ${ }^{15} \mathrm{EtG}$ is also a direct metabolite of ethanol which is formed in the liver and deposited into the hair follicles following the consumption of alcohol. The cut-off for EtG to indicate frequent excessive alcohol consumption is $30 \mathrm{pg} / \mathrm{mg} .{ }^{15}$

\section{Hair analysis for drugs}

Hair analysis was used to test for amphetamine, cannabis, cocaine, ecstasy, methadone, methamphetamine and opiates. The following cut-offs were used: $3.0 \mathrm{ng} / \mathrm{mg}$ for amphetamine, $5.0 \mathrm{ng} / \mathrm{mg}$ for cocaine, $3.0 \mathrm{ng} / \mathrm{mg}$ for ecstasy, $5.0 \mathrm{ng} / \mathrm{mg}$ for methadone, $3.0 \mathrm{ng} / \mathrm{mg}$ for methamphetamine and $2.0 \mathrm{ng} / \mathrm{mg}$ for opiates.

\section{Data analysis}

Data were analysed using SPSS Version 18. Sensitivity, specificity, and positive and negative predictive values were determined (comparing self-report [DUDIT + AUDIT] with biological markers [in urine and hair]). Student's t-tests were conducted to assess gender differences in AUDIT and DUDIT scores. A two-tailed p $<0.05$ was considered statistically significant.

\section{Results}

\section{Demographic and clinical characteristics}

The majority of the sample consisted of Black and Coloured females, reflecting the demographics of the population of persons living with HIV attending the public health clinic where the data were collected (Table I). The terms "White", "Black", and

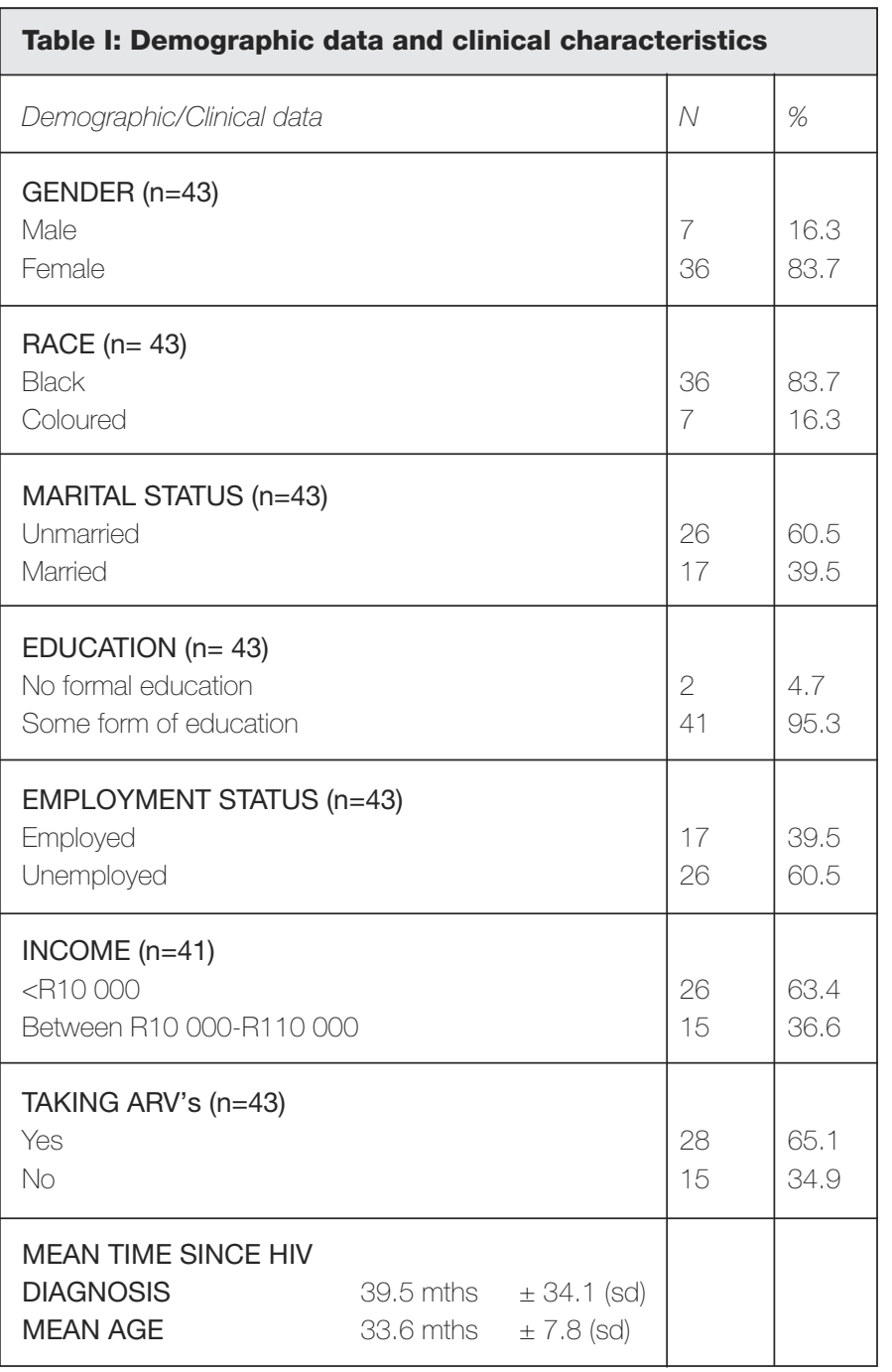


"Coloured" refer to demographic groupings used in South Africa and do not signify inherent characteristics. Their continued use in South Africa is important for monitoring improvements in health and socio-economic disparities, identifying vulnerable sections of the population, and planning effective prevention and intervention programmes. Most of the participants were poor, unemployed, and relatively young, with an average age of 34 years (23-59 years). The majority (65\%) were on ARVs at the time of data collection. The average time between diagnosis of HIV and data collection was 39.5 months (2 months-10 years).

\section{Comparison of self-reported measures of problematic} alcohol and drug use with urine and hair tests

All 43 participants completed the AUDIT. Of these, 18 (41.9\%) scored at or above the clinically significant cut-off score of 8 , indicating the likelihood of harmful and hazardous drinking. Of 30 participants tested for alcohol markers (FAEE and EtG) in hair, 2 (7\%) tested positive for alcohol abuse on FAEE and 6 (25\%) tested positive for alcohol abuse on EtG.

Of the 43 participants who completed the DUDIT, 12 (28\%) scored above the respective cut-off points on the DUDIT ( $\geq 2$ for females; $\geq 6$ for males) for over-the-counter drugs (OTC) and 1 tested positive for cannabis while none of the participants scored above the cut-off for other drugs. In contrast, using hair analysis, all 30 tested negative for cannabis, amphetamines, opiates, cocaine, PCP and methaqualone. Urinalysis revealed one positive test for cannabis which is consistent with the results of the DUDIT, with the remaining participants testing negative for amphetamines, opiates, cocaine, PCP and methaqualone.

Sensitivity and specificity analysis of the self-report measures versus the biological markers

A comparison of scores of AUDIT with FAEE and EtG is provided in Tables II a and II b. The sensitivity (TP/ (TP + FN) x 100) of the AUDIT against the FAEE and EtG to detect harmful and hazardous drinking in the participants was 100\% and $83.3 \%$ indicating that the AUDIT is good at not missing people who have an alcohol problem as indicated by the biological markers (i.e., high rate of true positives). The specificity (TN/ $(\mathrm{FP}+\mathrm{TN}) \mathrm{x} 100)$ of the AUDIT using the FAEE was $53.6 \%$ and $61.1 \%$ when using the EtG. Thus, the AUDIT is only "fair" at detecting people who do not have an alcohol problem (i.e., moderate rate of true negatives).

The positive predictive value (PPV) (TP/ (TP + FP x 100) for the AUDIT against the FAEE was 13\% and on the EtG was 41.6\%, indicating only a few of the participants who were screened positive were confirmed by the biological marker. The negative predictive value (NPV) (TN/(TN + FN) x 100) on the FAEE was $100 \%$ and on the $\mathrm{EtG}$ was $91.6 \%$, indicating that a high proportion of the participants who screened negative were in fact negative.

A comparison of scores of the DUDIT with the biological marker (hair) is provided in Table IV. The sensitivity, specificity, PPV and NPV of the DUDIT against the biomarker (hair) to detect harmful use or dependence of drugs in the participants were 0\%,70\%, 0\% and 100\%, respectively. A comparison of scores of the DUDIT with the biological marker (urine) is provided in Table IV. The sensitivity, specificity, PPV and NPV of the DUDIT against biomarkers in the urine to detect harmful use or dependence of drugs in the participants were 100\%, $66 \%, 10 \%$ and $100 \%$, respectively.

\section{Discussion}

The accuracy of the AUDIT was very high compared to a biological marker (hair) in the detection of an alcohol-related problem, but was only moderately good in detecting people who did not have an alcohol related problem. All participants, on both the DUDIT and hair testing, screened negative for a drug-related problem. The accuracy of the DUDIT was very high compared to urine analysis in the detection of a drug-

Table II a: Hair alcohol biomarkers versus AUDIT

FAEE

\begin{tabular}{|c|c|c|}
\hline - & $\begin{array}{c}\text { True negative (TN) } \\
15\end{array}$ & $\begin{array}{c}\text { False negative (FN) } \\
0\end{array}$ \\
\hline+ & $\begin{array}{c}\text { False positive (FP) } \\
13\end{array}$ & $\begin{array}{c}\text { True positive (TP) } \\
2\end{array}$ \\
\hline & 28 & 2 \\
\hline
\end{tabular}

\section{Table II b}

EtG

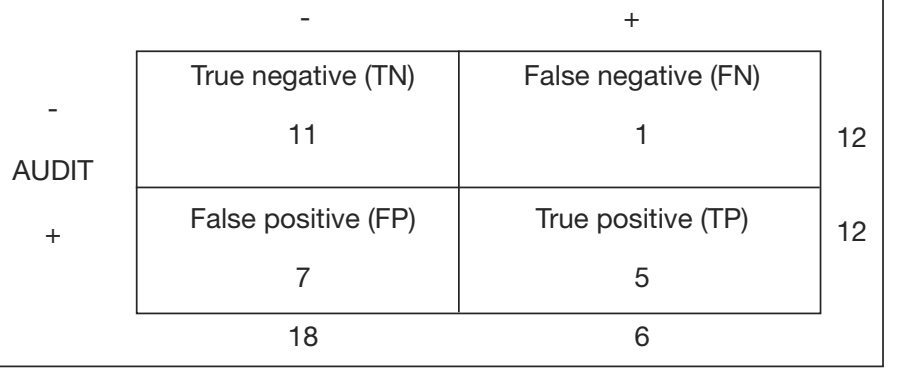

Table III: Hair drug biomarker versus DUDIT

EtG

\begin{tabular}{|c|c|c|c|}
\hline - & $\begin{array}{c}\text { True negative (TN) } \\
21\end{array}$ & $\begin{array}{c}\text { False negative (FN) } \\
0\end{array}$ & $\begin{array}{r}\begin{array}{r}\text { Hair drug } \\
\text { biomarker }\end{array} \\
21\end{array}$ \\
\hline+ & $\begin{array}{c}\text { False positive (FP) } \\
9\end{array}$ & $\begin{array}{c}\text { True positive (TP) } \\
0\end{array}$ & 9 \\
\hline & 30 & 0 & \\
\hline
\end{tabular}

Table IV: Urine drug analysis versus DUDIT

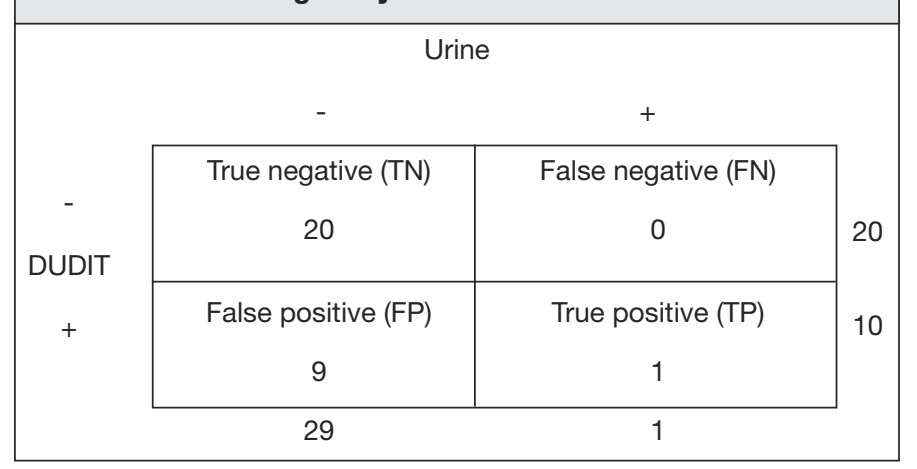


related problem. Thus, these self-report measures are not likely to indicate that someone has a severe substance abuse problem if they do not have one. However, the selfreport measures tended to over predict the likelihood of a person having a substance related problem if hair and urine testing are used as the gold standard.

The discrepancy between biological hair analysis and self-report (AUDIT \& DUDIT) findings may relate to the timing of substance use. Biological markers are able to detect use over a relatively short time period only (for hair: up to 3 months given the length of hair we obtained; for urine: maximum of two weeks and a minimum of 72 hours depending on the drug of use), while the AUDIT and DUDIT measure alcohol and drug use over a longer time frame (i.e. up to a year). Differences in time measurement may explain why the AUDIT and DUDIT detected higher rates of alcohol and drug use among participants.

The findings of this study like many studies have found that there is a high rate of substance abuse among people who are HIV positive 6 19,20 In South Africa a study conducted on HIV patients attending an infectious diseases clinic at Tygerberg Hospital in Cape Town in revealed that 10.1\% of the patients were alcohol dependent. ${ }^{7}$ A cross-sectional study also conducted in Cape Town between October 2004 and December 2005 found that of the 465 HIV positive patients enrolled for HIV treatment and care at primary health care services, 7\% reported alcohol

dependence/abuse. ${ }^{8}$

These findings are also consistent with a study ${ }^{20}$ conducted in the emergency department of a university hospital in Germany that found the AUDIT to be superior in detecting alcohol abuse in comparison to biomarkers such as Gamma-Glutamyl-Transferase (GGT), Mean Corpuscular Volume (MCV) and Carbohydrate-Deficient-Transferrins (GGT). However, a study ${ }^{16}$ conducted in Britain on parents with suspicious alcohol abuse in child protection cases that compared self-reported drinking data with FAEE showed that FAEE has a longer time period of detection than CDT and GCT and hence has increased sensitivity and specificity.

In terms of practicalities, obtaining hair samples from Black and Coloured participants proved to be challenging. In many instances adequate hair samples were not obtained as hair was too short (less than $1 \mathrm{~cm}$ ) and curly. In addition, most women had their hair plaited in braids which resulted in an inability to acquire a natural strand of head hair. For successful analysis of hair samples, it is recommended that the hair strand be at least 3-6 cm long. ${ }^{16}$

Furthermore, the cost of doing both urine and hair assays was very high in comparison with self-report measures. Hair assays cost approximately Rl 593 (\$212) per participant and the urine test cost R1 180 (\$157) per participant. Urine tests were sent away for analysis as opposed to the dip-stick approach which is cheaper and gives an immediate result but is subject to other limitations (reference).

Hair biomarkers although costly can be useful and beneficial in primary health care settings in assisting health care professionals in the early identification of patients with substance abuse problems. Biomarkers can give important objective information regarding the severity of the substance abuse problem, help with diagnosis and assist in the monitoring of alcohol related medical conditions. Biomarkers could also serve as an objective measure of substance abuse treatment outcomes and provide patients with feedback about the effects of drugs and alcohol on their condition. Primary health care settings could benefit from these tests since early detection of substance abuse could result in considerable savings in medical costs and lessen the burden in primary health care settings where patients have alcohol and drugs problems that exacerbate their other medical conditions. ${ }^{21}$

However, considering the high cost and low detection rate, it is probably not cost-effective to conduct biological testing for substance use in resource poor settings. Such tests are also much more invasive than self-report measures. Self-report measures had high sensitivity compared to the biomarkers and seemed to identify a wider range of HIV patients having possible substance use problems.

The AUDIT and DUDIT are two screening instruments that can be utilised in all treatment settings to screen for alcohol and drug use by health workers with varying levels of training and experience. ${ }^{14,18}$

While both these instruments are not used routinely to screen for alcohol and drug use problems in primary health care clinics in South Africa, they are becoming increasingly recognised by clinicians as useful tools for the detection of alcohol and drug use. ${ }^{22}$

\section{Study limitations}

Study limitations include the small sample size and localisation of the study to one HIV clinic which makes generalisation of findings to other settings and populations difficult. However, it should be noted that this was a pilot study with the primary purpose of evaluating biomarker assay methods and their feasibility and cost. The sample, therefore, represents approximately $5 \%$ of the sample to be recruited in a larger study of HIV, mental disorder, and substance use comorbidity that followed the pilot study. Despite the limitations, the preliminary findings are important because it is the first study undertaken in a South African population of HIV clinic attendees to investigate the use of self-report measures (AUDIT and DUDIT) and biological measures (hair and urine) in the detection of alcohol and drug use.

\section{Conclusions}

This study highlights the problem of substance abuse among patients attending HIV clinics, especially abuse of alcohol. Furthermore, the findings emphasise the importance of screening HIV patients for substance abuse and dependence. Addressing alcohol and drug related problems should be standard protocol for ARV treatment programmes for patients attending HIV clinics. ${ }^{23}$ Similarly, health workers working in the field of substance abuse need to consider including HIV intervention services into their programmes. ${ }^{24}$ Self-report measures (AUDIT and DUDIT) are a more viable option for screening of alcohol and drug abuse in resource poor settings than biological markers. Furthermore, combining biological markers with self-report measures does not appear to add much value to these questionnaires used on their own. 


\section{Acknowledgements}

The authors wish to acknowledge the staff at Wallacedene clinic for their assistance in helping recruit participants for this study, the administration at the Provincial Department of Health, the patients who participated in this study and the research team (Ms Lewina Rowland and Ms Siphokazi Dada) for their fieldwork and data entry. The MRC would also like to acknowledge Trimega Laboratories for making the hair kits available at a cost price.

\section{References}

1. Statistics South Africa: Mid-year population estimates 2009. http://www.statssa.gov.za/publications/P0302/P03022010.pdf (accessed 19 October 2010).

2. Parry CDH, Pithey AL. Risk behaviour and HIV among drug using populations in South Africa. African Journal of Drug and Alcohol Studies 2006; 5 (2):140-157.

3. Stein J, Seedat S, Herman AA, Heeringa SG, Moomal H, Myer L, Williams D. Findings from the first South African stress and health study. Policy Brief. South African Medical Research Council. 2007.

4. Peltzer K, Davids A, Njuho. Alcohol use and problem drinking in South Africa: findings from a national population-based survey. African Journal of Psychiatry 2011;14: 30-37.

5. Hendershot CS, Stoner SA, Pantalone, DW, Simoni, JM. Alcohol use and antiretroviral adherence: review and meta-analysis. Journal of Acquired Immnune Deficiency Syndrome 2009; 52:180-202.

6. Cook RL, Sereika, SM, Hunt WC, Woodward CW, Erlen JA, Conigliaro J. Problem drinking and medication adherence among persons with HIV infection. Journal of General Internal Medicine 2001; 16: 83-88.

7. Olley BO, Gxama F, Seedat S, Theron H, Taljaard J. Reid E, Reuter H, Stein DJ. Psychopathology and coping in recently diagnosed HIVIAIDS patients-the role of gender. South African Medical Journal 2003; 93 (12): 928-931

8. Myer L, Smit J, Le Roux, L, Parker, S, Stein, D, Seedat S. Common mental disorders among HIV-infected individuals in South Africa: Prevalence, predictors, and validation of brief psychiatric rating scales. AIDS Patient Care and STDs 2008; 22 (2):147-158

9. Plüddemann A, Parry CDH. A short report: self-reported drug use vs. urinalysis in a sample of arrestees in South Africa. Drugs: Education, Prevention and Policy 2003; 10 (4): 379-383.

10. Gorman ME, Carroll RT. Substance abuse and HIV: Considerations with regard to methamphetamines and other recreational drugs for nursing practice and research. Journal of the Association of Nurses in AIDS Care 2000; 11 (2): 51-62.

11. Shuper PA, Neuman M, Kanteres F, Baliunas D, Joharchi N, Rehm J. Causal considerations on Alcohol and HIVIAIDS - A systematic review. Alcohol and Alcoholism 2010; 1-8.

12. Niemela O. Biomarkers in alcoholism. Clinica Chimica Acta 2007; 377: $39-49$
13. Siegfried N, Parry CDH Morojele NK, Wason D. Profile of drinking behavior and comparison of self-report with the CAGE questionnaire and carbohydrate-deficient transferring in a rural Lesotho community. Alcohol \& Alcoholism 2001; 36 (3): 243-248.

14. Babor TF, Higgins-Biddle JC, Saunders JB, Monteiro MG. AUDIT. The Alcohol Use Disorders Identification Test: Guidelines for use in primary care. Second edition. World Health Organization. Department of Mental Health and Substance Dependence 2001.

15. Pragst F, Yegels M. Determination of fatty acid ethyl esters (FAEE) and ethyl glucuronide (EtG) in hair: A promising way for retrospective detection of alcohol abuse during pregnancy? Ther Drug Monit 2008; 30: 255-263.

16. Süße S, Selavka CM, Mieczkowski T, Pragst, F. Fatty acid ethyl ester concentrations in hair and self-reported alcohol consumption in 644 cases from different origin. Forensic Science International 2010; 196. $111-117$

17. Sheehan DV, Lecrubier Y, Hairnet-Sheehan K, Amorin P, Janavs J, Weiler E, et al. The MINI-International Neuropsychiatric Interview (MINI): the development and validation of a structured diagnostic psychiatric interview. Journal of Clinical Psychiatry 1998; 59 (20):2233

18. Berman AH, Bergman H, Palmstierna T, Schlyter F. DUDIT Manual. The Drug Use Disorders Identification Test. Karolinska Institute, Department of Clinical Neuroscience; 2005.

19. Mellins CA, Kang E, Leu CS, Havens JF, Chesney MA. Longitudinal study of mental health and psychosocial predictors of medical treatment adherence in mothers living with HIV disease. AIDS Patient Care and STDs 2003; 17 (8): 407-416.

20. Palepu A, Khan NA, Norena M, Wong H, Chittock DR, Dodek PM. The role of HIV infection and drug and alcohol dependence in hospital mortality among critically ill patients. Journal of Critical Care 2008; 23 (3):275-280

21. Litten RZ, Bradley AM, Moss HB. Alcohol biomarkers in applied settings: Recent advances and future research opportunities. Alcoholism: Clinical and Experimental Research 2010; 34 (6): 955967.

22. Koopman FA, Parry CDH, Myers B, Reagon G. Adressing alcohol problems in primary care settings: A study of general medical practitioners in Cape Town, South Africa. Scandinavian Journal of Public Health 2008; 36: 298-302.

23. NeumanT, Gentilello LM, Neuner B, Weib-Gerlach E, Schürmann H, Schröder T, Müller C, Haas NP, Spies CD. Screening trauma patients with the Alcohol Use Disorders Identification Test and biomarkers of alcohol use. Alcoholism: Clinical and Experimental Research 2009; 33 (6):970-976

24. Morojele, NK, Pithey, AL, Pule MI, Joubert M. Alcohol and HIV: Research, programmes and resources in South Africa. Report to the US Department of State. Alcohol and Drug Abuse Research Unit, Medical Research Council; 2006.

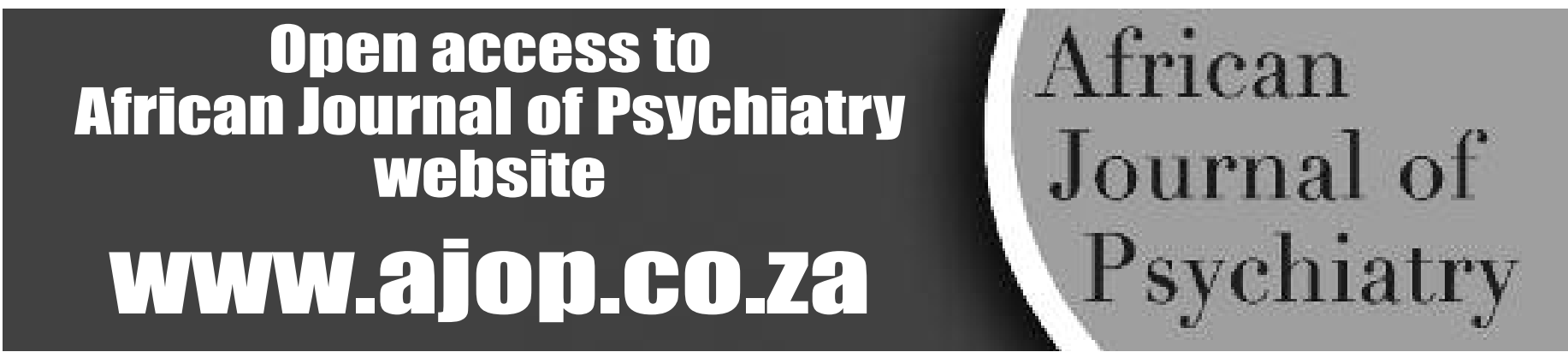

